\title{
Ambient Illumination as a Context for Video Bit Rate Adaptation Decision Taking
}

\author{
G. Nur, H. Kodikara Arachchi, Member, IEEE, S. Dogan, Member, IEEE, and A. M. Kondoz, \\ Member, IEEE
}

\begin{abstract}
In this letter, a new bit rate adaptation decision taking technique is proposed based on the observations that ambient illumination context has an effect on the perceived video quality. Motion activity and structural complexity characteristics of a video content are utilized as the content related contexts in the proposed technique. Experimental results demonstrate that a significant amount of bit rate can be saved by adapting the video content according to different ambient illumination conditions using this technique. Subjective assessment results show that the proposed adaptation technique is capable of exploiting the changes in ambient illumination level for bit rate adaptation without sacrificing the perceived visual quality.
\end{abstract}

Index Terms - Adaptation decision taking, ambient illumination, motion activity, structural complexity.

\section{INTRODUCTION}

Universal Multimedia Experience (UME) is a key concept that has a vision for achieving improved quality of viewing experiences [1][2]. Adaptation of video content is a strategic field under the umbrella of this vision [3][4]. The overall target of video adaptation is to maximize the user experience in terms of perceived quality. Adaptation decision taking, which aims to determine optimum parameters for adaptation operations, can be considered as the "brain" of video content adaptation. Therefore, efficient adaptation decision taking techniques should be developed to accomplish the aforementioned target of video adaptation.

Context-awareness plays a significant role in adaptation decision taking for achieving enhanced quality of viewer experiences [5]. Content-related contexts, such as motion activity and structural complexity of video, as well as external contexts, such as viewing environment, may influence the adaptation decisions [6]. Using ambient illumination condition as a context during decision taking is an interesting research topic, which is yet to be thoroughly investigated for content adaptation purposes. Ambient illumination context can be gathered through light sensors placed on user devices to collect information about the level of brightness in the consumption environment.

Manuscript received ...., 2010...

Gokce Nur, Hemantha Kodikara Arachchi, Safak Dogan, and Ahmet M. Kondoz are with the I-Lab Multimedia Communications Research, Faculty of Engineering and Physical Sciences, University of Surrey, Guildford, GU2 7XH, Surrey, UK. (phone: +44-(0)1483-684731; fax: +44-(0)1483-686011; e-mail: g.nur@surrey.ac.uk).
The main goal of the work presented in this letter is to devise a new video bit rate adaptation decision taking technique, which relies on deriving decisions based on the ambient illumination. To achieve this goal, first the perceived video quality is subjectively assessed under different ambient illumination conditions. Exploiting the knowledge gained through the initial subjective experiments, the proposed technique is developed. Video information is then adapted considering different ambient illumination conditions using the proposed technique. In order to assess the resulting quality of the adapted video content, further subjective experiments are conducted.

The rest of the letter is organized as follows. In Section II, the initial subjective assessments are presented. Subjective assessment results and observations about these results are introduced in Section III. In Section IV, the proposed adaptation decision taking technique is discussed. The adaptation decision taking results are provided and assessed in Section V. Finally, Section VII concludes the letter.

\section{INITIAL SUBJECTIVE ASSESSMENTS}

In order to assess the effect of ambient illumination on the perceived video quality to devise an adaptation decision taking technique, a number of initial subjective experiments were conducted. Nine test segments were used in the experiments rather than the entire test sequences to maintain homogeneity in the motion activity and structural complexity characteristics for a certain length in the test contents. The name of the nine test segments are: Stefan, Football, Soccer, City, Tempete, Flower Garden, News, Weather Forecast, and Silent [7], and they contain the frames: 10-160, 0-150, 30-180, 0-150, 0-150, $5-155,0-150,0-150$, and $0-150$ of their respective sequences. The structural complexity and motion activity are referred to as $C$ and $M$ in the letter, respectively.

The test segments were of CIF (352×288 pels) spatial resolution at $30 \mathrm{fps}$. Eight different channel bandwidths (i.e., $64,96,128,192,256,384,512$, and $768 \mathrm{kbps})$ were selected as the target channel rates for test purposes. The segments were encoded using the Joint Scalable Video Model (JSVM) reference software version 9.13.1 [8]. A 23" Dell display, which has a resolution of 1680x1050 pels, was used to display the sequences. The Double Stimulus Continuous Quality Scale (DSCQS) method [9] was used throughout the tests. DSCQS was chosen in order to assess the quality of the encoded content with respect to the maximum quality that can be offered to the user (i.e., the original video sequence). 16 viewers (10 experts and 6 non-experts) participated in the experiments. The effect of the ambient illumination on 
perceptual quality was assessed in four different ambient illumination conditions (i.e., 2, 53, 195, and 293 lux) created by our laboratory facilities, in which the experiments were conducted. These conditions were measured using a Gretag Macbeth Eye-One Display 2 device [10]. Ambient illumination is referred to as $I$ in this letter. The lengths of the videos were restricted to 5 seconds, which is in compliance with the International Telecommunication Union (ITU)'s recommendation [9]. The subjective tests were conducted for each viewer to assess all of the test segments individually, which were randomly ordered for each environment condition to prevent any prejudice. After the experiments, the Mean Opinion Scores (MOSs) [9] obtained from all of the viewers were computed.

\section{INITIAL SUBJECTIVE ASSESSMENT RESULTS AND DISCUSSION}

The initial subjective test results are discussed in this section for the video segments in view, which correspond to different $M$ s ranging from high to low. Fig. 1 illustrates the experimental results for the test segments. As can be observed from the figures, the curves representing the MOSs recorded in the 2 lux environment present the least favorable perceived quality reported by the viewers. The 53, 195, and 293 lux environments demonstrate an increasing order of MOS ratings. The reason is that in the 2 lux (i.e., dark) environment, the sensitivity of the Human Visual System (HVS) is greater, because the iris enlarges in response to the less amount of ambient light enters into the eye. As a result, in such an environment, the compression artifacts in a visual scene are more distinguishable to the eye than in the other environments.

As can be seen in Fig. 1 (a), the point marked as A on the 53 lux curve and the point marked as B on the 2 lux curve result in similar MOSs despite corresponding to different bit rates. Similar behavior can also be observed for the other points on the other curves. The observation is that when $I$ increases (e.g., from 2 to 53 lux), the perceived quality of an input video segment is not compromised by viewing it at a lower bit rate. In this way, the bit rate of the video segment can be reduced by a significant amount if viewing takes place in a brighter environment. The change in the $I$ condition is referred to as $\Delta I$ and three different $\Delta I$ s are considered: 2-53, 2-195, and 2-293 lux in this Letter. The principle idea here is that the input video segment can be adapted to a lower bit rate without causing any compromise in the perceived video quality under a particular $\Delta I$. Finding the output bit rate of the adapted video under a $\Delta I$ is the main aim of the proposed technique. In order to achieve this aim, the mathematical functions of every curve in the graphs have been determined, as illustrated in the figures. The generic form of a function of any curve is given in (1):

$$
M O S=K \ln (B)+L
$$

where $B$ is the bit rate, $K$ and $L$ are the constants. Table I shows the $K$ and $L$ values of the functions of the different $I$ condition curves for the different segments. As seen from the table, one of the factors that the values of the constants depend

on is $I$. As can also be noticed, the values change for different Ms. However, News, Silent and Weather Forecast sequences result in very similar $M$ s (i.e., 0.096, 0.095, and 0.094) even if the constant values vary for the same $I$ conditions. Therefore, it is clear that $M$ is not the only content related characteristic that determines the values. It has been derived after extensive analyses that $C$ is the other content related context that has an effect on the values. The reason why $C$ is also important in determining the values is that HVS is typically used to extracting structural information from a visual scene rather than the errors in the scene [11]. Concisely, after the extensive analyses, it has been found that $I, M$, and $C$ are the dominant factors that determine the values of the constants.

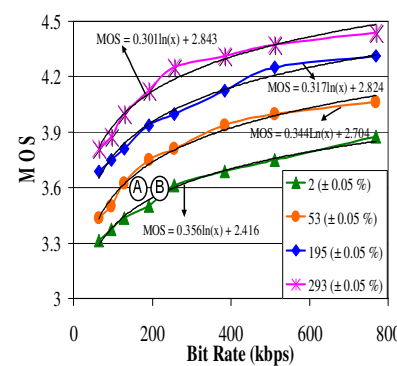

(a)

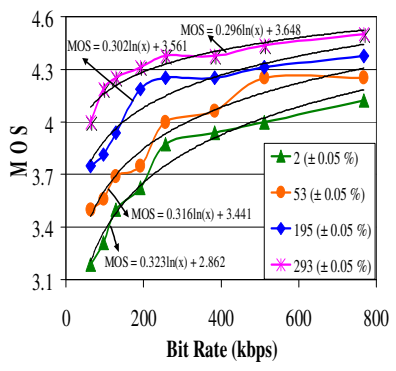

(c)

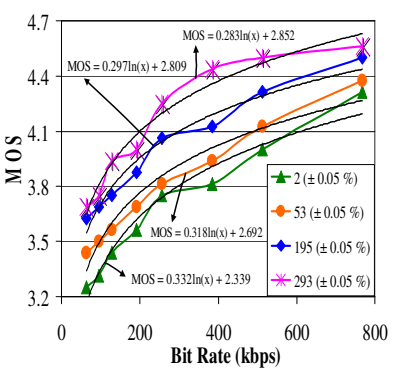

(e)

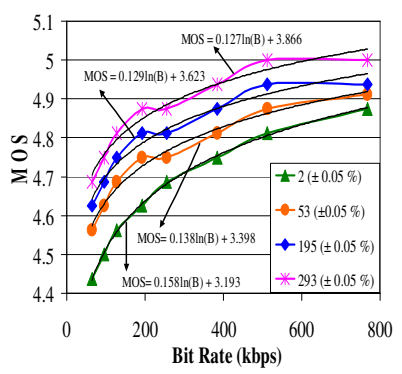

(g)

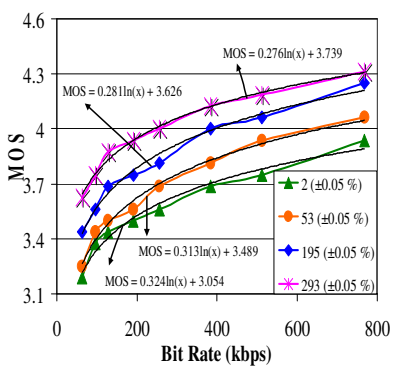

(b)

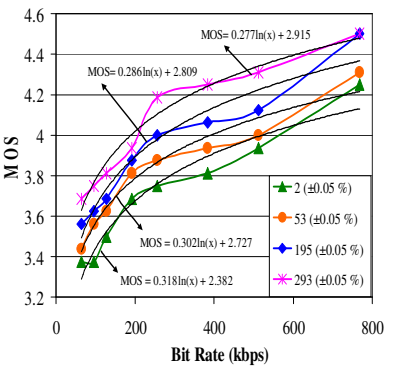

(d)

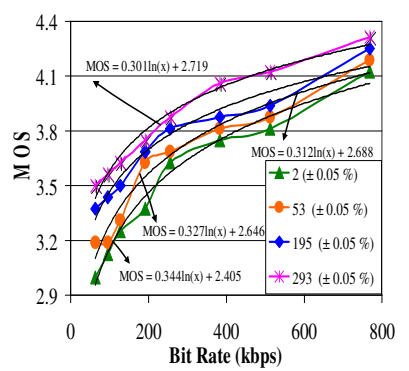

(f)

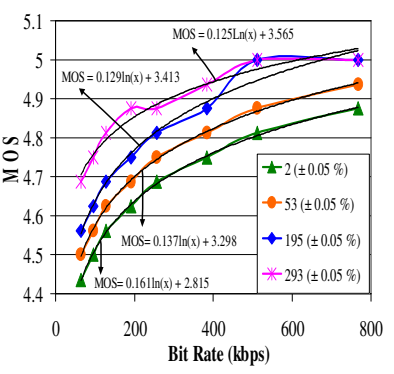

(h) 


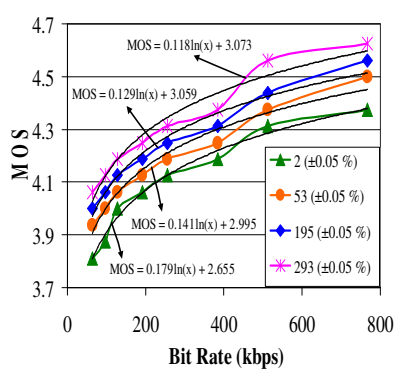

(i)

Fig. 1 MOS scores at various bit rates under different $I$ conditions for (i) high motion: (a) Stefan (b) Football (c) Soccer; (ii) medium motion: (d) City (e) Tempete (f) Flower Garden; and (iii) low motion: (g) News (h) Weather Forecast (i) Silent, respectively

TABLE I

EXPERIMENTAL AND CALCULATED CONSTANTS FOR THE VIDEO SEGMENTS VIEWED UNDER DIFFERENT IS

\begin{tabular}{|c|c|c|c|c|c|c|c|}
\hline \multirow{2}{*}{ Segment } & \multirow{2}{*}{$M$} & \multirow{2}{*}{$C$} & \multirow{2}{*}{$I$} & \multicolumn{2}{|c|}{ Experimental } & \multicolumn{2}{|c|}{ Calculated } \\
\hline & & & & $K$ & $L$ & $K$ & $L$ \\
\hline \multirow{4}{*}{ Stefan } & \multirow{4}{*}{0.412} & \multirow{4}{*}{0.199} & 2 & 0.356 & 2.416 & 0.353 & 2.392 \\
\hline & & & 53 & 0.344 & 2.704 & 0.328 & 2.682 \\
\hline & & & 195 & 0.317 & 2.824 & 0.326 & 2.758 \\
\hline & & & 293 & 0.301 & 2.843 & 0.325 & 2.780 \\
\hline \multirow{4}{*}{ Football } & \multirow{4}{*}{0.718} & \multirow{4}{*}{0.098} & 2 & 0.324 & 3.054 & 0.288 & 3.095 \\
\hline & & & 53 & 0.313 & 3.489 & 0.266 & 3.684 \\
\hline & & & 195 & 0.281 & 3.626 & 0.264 & 3.838 \\
\hline & & & 293 & 0.276 & 3.739 & 0.263 & 3.882 \\
\hline \multirow{4}{*}{ Soccer } & \multirow{4}{*}{0.512} & \multirow{4}{*}{0.085} & 2 & 0.323 & 2.862 & 0.317 & 2.748 \\
\hline & & & 53 & 0.316 & 3.441 & 0.295 & 3.376 \\
\hline & & & 195 & 0.302 & 3.561 & 0.293 & 3.509 \\
\hline & & & 293 & 0.296 & 3.648 & 0.289 & 3.587 \\
\hline \multirow{4}{*}{ City } & \multirow{4}{*}{0.241} & \multirow{4}{*}{0.171} & 2 & 0.318 & 2.382 & 0.293 & 2.298 \\
\hline & & & 53 & 0.302 & 2.727 & 0.269 & 2.632 \\
\hline & & & 195 & 0.286 & 2.809 & 0.267 & 2.719 \\
\hline & & & 293 & 0.277 & 2.915 & 0.263 & 2.744 \\
\hline \multirow{4}{*}{ Tempete } & \multirow{4}{*}{0.217} & \multirow{4}{*}{0.207} & 2 & 0.332 & 2.339 & 0.287 & 2.248 \\
\hline & & & 53 & 0.318 & 2.692 & 0.264 & 2.527 \\
\hline & & & 195 & 0.297 & 2.809 & 0.261 & 2.600 \\
\hline & & & 293 & 0.283 & 2.852 & 0.258 & 2.621 \\
\hline \multirow{4}{*}{$\begin{array}{l}\text { Flower } \\
\text { Garden }\end{array}$} & \multirow{4}{*}{0.266} & \multirow{4}{*}{0.194} & 2 & 0.344 & 2.405 & 0.312 & 2.285 \\
\hline & & & 53 & 0.327 & 2.646 & 0.288 & 2.582 \\
\hline & & & 195 & 0.312 & 2.688 & 0.286 & 2.660 \\
\hline & & & 293 & 0.301 & 2.719 & 0.285 & 2.683 \\
\hline \multirow{4}{*}{ News } & \multirow{4}{*}{0.096} & & 2 & 0.158 & 3.193 & 0.141 & 2.868 \\
\hline & & 0.074 & 53 & 0.138 & 3.398 & 0.119 & 3.348 \\
\hline & & & 195 & 0.129 & 3.623 & 0.117 & 3.553 \\
\hline & & & 293 & 0.127 & 3.866 & 0.116 & 3.611 \\
\hline & & & 2 & 0.161 & 2.815 & 0.138 & 2.501 \\
\hline Weather & 0.094 & 0.084 & 53 & 0.137 & 3.298 & 0.116 & 3.189 \\
\hline Forecast & & & 195 & 0.129 & 3.413 & 0.114 & 3.369 \\
\hline & & & 293 & 0.125 & 3.565 & 0.113 & 3.420 \\
\hline & & & 2 & 0.179 & 2.655 & 0.146 & 2.302 \\
\hline Silent & 0.095 & 0.141 & 53 & 0.141 & 2.995 & 0.121 & 2.711 \\
\hline & & & 195 & 0.129 & 3.059 & 0.118 & 2.818 \\
\hline & & & 293 & 0.118 & 3.073 & 0.115 & 2.849 \\
\hline
\end{tabular}

\section{PROPOSED ADAPTATION DECISION TAKING TECHNIQUE}

The observations from the initial tests have indicated that an adaptation decision taking technique needs to consider three factors: the $I$ condition, the $M$, and the $C$ of the input video for efficient adaptation to produce the target output video bit rate.

The $M$ of a segment is measured using its motion intensity.
The optical flow algorithm of Lucas \& Kanade [12] is used for motion intensity measurements. The motion intensities of several segments with different spatio-temporal resolutions were analyzed using this algorithm. It has been observed that these resolutions should be normalized to allow for consistency across different segments. The mathematical model presented in (2) is devised for performing the $M$ measurement:

$$
M=\frac{\sum_{n=1}^{\mathrm{NoF}} \Pi(n)}{\mathrm{NoF}} \cdot \frac{F}{S}
$$

where $\Pi(n)$ is the motion intensity of the $n^{\text {th }}$ frame of a segment. NoF is the number of frames in the segment. $F$ and $S$ are the frame rate and spatial resolutions of the segment, respectively.

Contours, which characterize the boundaries of the objects in video frames, are used to represent the $C$ of the visual scenes in this letter. Canny edge detection algorithm [8] was used to determine the contours in the frames without suppressing the pixels that are considered as edges by setting them to 1 [13]. To develop the structural complexity algorithm, the number of pixels that are set to 1 is counted in every frame of a video segment [14][15][16]. The total value is then normalized using the NoF and $S$ to provide consistency across different video segments as follows:

$$
C=\frac{\sum_{n=1}^{\mathrm{NoF}} \delta(n)}{\operatorname{NoF} S}
$$

where $\delta(n)$ is the number of edge pixels in the $n^{\text {th }}$ frame.

In order to devise generic functions for $K$ and $L$ in (1) while developing the proposed technique, firstly, the graphs representing the $C$ vs $K, M$ vs $K, I$ vs $K, C$ vs $L, M$ vs $L$, and $I$ vs $L$ are plotted, as illustrated in Fig. 2. Secondly, the relationships between all of these pairs are approximated using curve fitting functions [17], as also illustrated in Fig. 2. The constants in the functions are presented with $P, U, V, Y, G, J$, $D, E, H, R$, and $T$ in the figure.

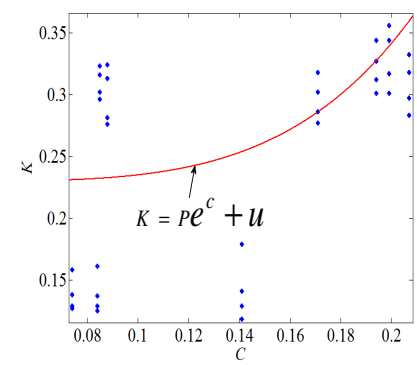

(a)

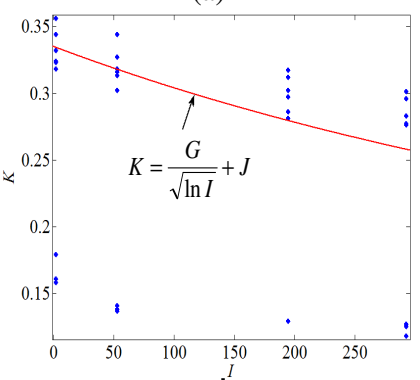

(c)

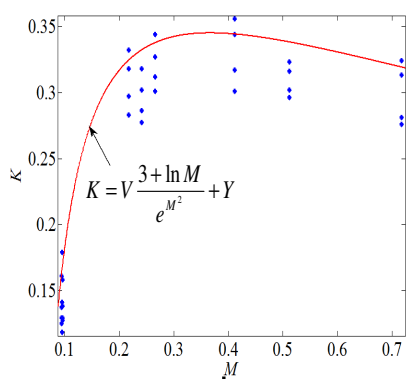

(b)

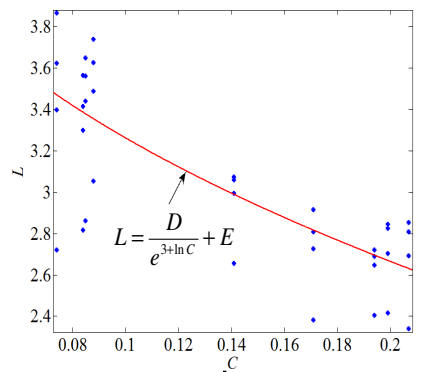

(d) 


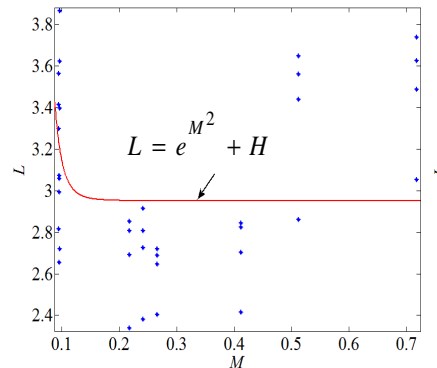

(e)

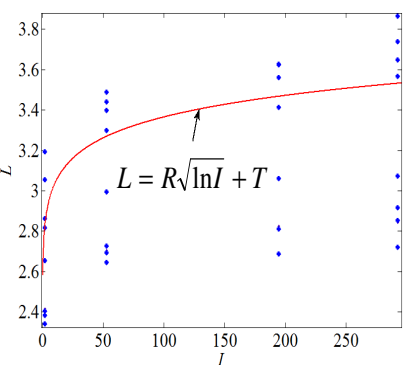

(f)
Fig. $2 K$ versus (a) $C$ (b) $M$ (c) $I$; and $L$ versus (d) $C$ (e) $M$ (f) $I$

Following, each of the $K$ and $L$ functions are empirically integrated together to determine the resulting equations that best correlate with the experimental $K$ and $L$. This has led to introducing a set of new numerical constants (i.e., 7, 0.2, and 1) to the equations while providing the best correlations. Accordingly, the generic functions of $K$ and $L$ are devised as follows:

$$
K=\frac{e^{C}}{7}\left(\frac{3+\ln M}{e^{M^{2}}}+\frac{0.2}{\sqrt{\ln I}}\right) \quad L=e^{M^{2}}+\frac{\sqrt{\ln I}}{e^{3+\ln C}}+1
$$

Table 1 also shows the $K$ and $L$ values calculated using the equation presented in (4) for the segments in view. The correlation coefficients computed between the values observed using the initial subjective experiments and the ones that are calculated for $K$ and $L$ are 0.985 and 0.961 , respectively.

To further describe the derivation of the proposed adaptation decision taking technique, a use-case scenario, in which a user is viewing a video segment in an environment, where $I=$ A lux, is considered. While continuing to watch the segment, s/he moves to a brighter environment, where $I=\mathrm{B}$ lux. In order to utilize the shared network resources better, the video segment can be adapted to a lower bit rate under B while maintaining the same perceptual quality as follows:

$$
\operatorname{MOS}_{a}=M O S_{i}
$$

where $a$ and $i$ represent the output adapted video segment, which is viewed under $B$, and the input video segment to be adapted, which is viewed under A, respectively. From (1) and (5), the proposed adaptation decision taking technique is devised to solve the following equation:

$$
\begin{gathered}
K_{a} \ln \left(B_{a}\right)+L_{a}=K_{i} \ln \left(B_{i}\right)+L_{i} \\
B_{a}=e^{\frac{K_{i} \ln \left(B_{i}\right)+L_{i}-L_{a}}{K_{a}}}
\end{gathered}
$$

where $K_{i}$ and $L_{i}$ are the constants for the video segment to be adapted, and $K_{a}$ and $L_{a}$ are the constants for the adapted video segment. (7) can be exploited to calculate the output bit rate $\left(B_{a}\right)$ for adapting an input video segment from a given input bit rate $\left(B_{i}\right)$ under a specified amount of $\Delta I$ without compromising the perceived video quality.

\section{RESULTS AND DISCUSSION}

In order to validate the efficiency of the proposed technique, adaptation decision taking experiments were carried out. The experiments were conducted with the test segments used in the initial subjective tests (i.e., Stefan, City, Flower Garden), together with three additional test segments (i.e., Tennis, Coastguard, Hall Monitor) considering the use-case scenario discussed in Section IV. The Is of the segments viewed in A and $\mathrm{B}$ conditions are referred to as $I_{\mathrm{i}}$ and $I_{\mathrm{a}}$, respectively. $I_{\mathrm{i}}$ was kept as 2 lux to maintain a common reference $I$ for all of the adaptation experiments whereas five different $I_{\mathrm{a}}$ s (i.e., 27 , 53, 102, 195, and 293 lux) were used in the experiments. The experiments were carried out for the input segments that have three different input bit rates (i.e., 384, 512, and $1024 \mathrm{kbps}$ ). Table II presents the resultant $B_{a}$ s using the input bit rates. To validate the resulting $B_{a}$ s for the adapted segments, further subjective experiments were conducted. The adapted segments were presented to the viewers under the aforementioned $I$ conditions. During the subjective evaluation tests, observers were asked to rate the video sequences according to the DSCQS method, as described in the ITU's recommendation on subjective quality assessments [9]. A five-level quality rating scale ranging from 1 (bad) to 5 (excellent) was used during the tests. 16 observers (10 experts and 6 non-experts) participated in the experiments. The MOS values were calculated for every video segment after the tests.

Fig. 3 shows the subjective test results of the adapted segments. As can be observed from the figure, the perceptual qualities of the adapted video segments either do not change or only slightly vary $(\sim 0.01 \%)$ under changing $I$ conditions. It can be argued that the proposed technique has been efficient to adapt the video segments while maintaining their respective perceptual qualities despite the different viewing $I$ conditions.

As mentioned earlier, the adaptation experiments were conducted considering the use-case scenario discussed in Section IV. The proposed technique is also capable of serving the adaptation needs in a reverse order operation in another use-case scenario, in which a user moves from a brighter environment into a darker one while watching a video segment. In this kind of a scenario, the output bit rate calculated by the proposed technique for the adapted video segment becomes higher than that for the input segment. Therefore, this adaptation is only feasible when necessary extra bandwidth is available. The reason of increased bit rate for the segment viewed in dark environment is that the compression artifacts are more visible to the eye in the darker environment than in the bright environment. In either of the scenarios, terminal functionalities for increasing or decreasing the brightness of the display can also be used for improving the perceived quality by enhancing the content visibility. However, it should be noted that our focus is not to improve the content visibility, but to redistribute shared network resources more effectively by exploiting the ambient illumination changes. 


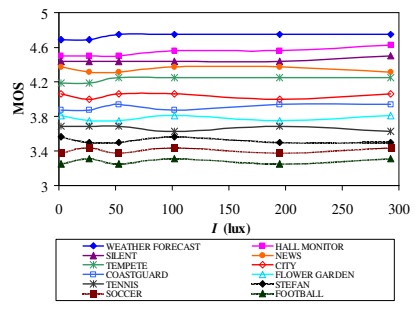

(a)

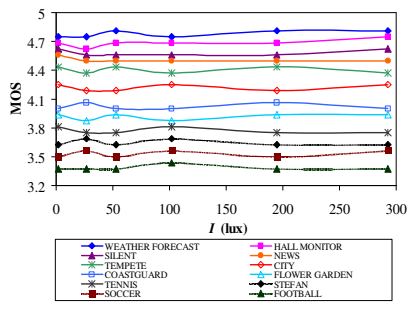

(b)

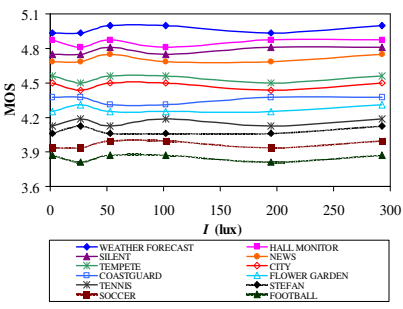

(c)

Fig. 3 MOS scores at (a) 384 (b) 512 and (c) 768 kbps under different $I$ conditions

TABLE II

RESULTING ADAPTED VIDEO BIT RATES USING THE PROPOSED TECHNIQUE

\begin{tabular}{|c|c|c|c|c|}
\hline \multirow{3}{*}{ Segment } & \multirow{3}{*}{$I_{a}(\operatorname{lux})$} & \multirow{2}{*}{\multicolumn{3}{|c|}{$B_{a}(\mathrm{kbps})$}} \\
\hline & & & & \\
\hline & & $B_{i}=384$ & $B_{i}=512$ & $B_{i}=1024$ \\
\hline \multirow{5}{*}{ Tennis } & 27 & 353.493 & 481.225 & 910.895 \\
\hline & 53 & 273.785 & 373.105 & 785.752 \\
\hline & 102 & 218.282 & 297.783 & 628.720 \\
\hline & 195 & 179.933 & 245.991 & 522.046 \\
\hline & 293 & 159.585 & 218.408 & 464.712 \\
\hline \multirow{5}{*}{ Coastguard } & 27 & 256.054 & 349.533 & 739.111 \\
\hline & 53 & 227.144 & 310.751 & 660.591 \\
\hline & 102 & 200.226 & 274.231 & 584.517 \\
\hline & 195 & 178.868 & 245.253 & 524.161 \\
\hline & 293 & 167.844 & 230.397 & 493.749 \\
\hline \multirow{5}{*}{$\begin{array}{c}\text { Hall } \\
\text { Monitor }\end{array}$} & 27 & 262.379 & 369.108 & 729.107 \\
\hline & 53 & 186.674 & 263.446 & 603.517 \\
\hline & 102 & 160.871 & 227.771 & 525.886 \\
\hline & 195 & 150.613 & 213.955 & 497.940 \\
\hline & 293 & 125.282 & 179.187 & 423.921 \\
\hline \multirow{5}{*}{ Stefan } & 27 & 276.356 & 376.053 & 789.147 \\
\hline & 53 & 249.332 & 339.918 & 716.551 \\
\hline & 102 & 225.069 & 307.132 & 648.914 \\
\hline & 195 & 204.290 & 279.041 & 590.918 \\
\hline & 293 & 194.070 & 265.337 & 563.196 \\
\hline \multirow{5}{*}{ City } & 27 & 218.806 & 298.728 & 631.889 \\
\hline & 53 & 188.420 & 257.839 & 548.446 \\
\hline & 102 & 168.618 & 231.012 & 492.768 \\
\hline & 195 & 141.472 & 194.050 & 415.102 \\
\hline & 293 & 138.709 & 191.176 & 413.710 \\
\hline \multirow{5}{*}{$\begin{array}{l}\text { Flower } \\
\text { Garden }\end{array}$} & 27 & 252.608 & 344.349 & 725.710 \\
\hline & 53 & 224.532 & 306.736 & 649.798 \\
\hline & 102 & 198.352 & 271.265 & 576.160 \\
\hline & 195 & 177.537 & 243.065 & 517.626 \\
\hline & 293 & 166.777 & 228.586 & 488.084 \\
\hline
\end{tabular}

\section{CONCLUSION}

A bit rate adaptation decision taking technique has been proposed based on the knowledge gained through subjective video quality assessments performed in different ambient illumination conditions. Structural complexity and motion characteristics of video have acted as the primary content related contexts that influence the adaptation decisions in this research work. The experiments demonstrated that a significant amount of bit rate can be saved under changing ambient illumination characteristics using the proposed technique. Subjective quality assessments proved the efficiency of this technique. The effects of other contexts that can be used in adaptation decision taking will be considered in future studies.

\section{REFERENCES}

[1] F. Pereira and I. Burnett, "Universal Multimedia Experiences for Tomorrow," IEEE Signal Process. Mag., vol. 20, no. 2, pp. 63-73, Mar. 2003.

[2] M. Waltl, C. Timmerer, H. Hellwagner, "A Test-bed For Quality of Multimedia Experience Evaluation of Sensory Effects," Proc.s of the First Int. Workshop on Quality of Multimedia Experience(QoMEX 2009), San Diego, USA, Jul., 2009.

[3] D. Mukherjee, E. Delfosse, J-G. Kim, Y. Wang, "Optimal Adaptation Decision Taking for Terminal and Network Quality of Service," IEEE Trans. Mult., vol. 7, no. 3, pp. 452-462, Jun. 2005.

[4] I. Kofler, C. Timmerer, H. Hellwagner, A. Hutter, and F. Sanahuja, "Efficient MPEG-21 Based Adaptation Decision Taking for Scalable Multimedia Content," Proc. $14^{\text {th }}$ Annual Elect. Imaging Conf, 2007.

[5] W.Y. Lum and F.C.M. Lau, "A Context-Aware Decision Engine for Content Adaptation," IEEE Pervasive Computing, vol. 1, no. 3, pp. 41-49, July-Sept. 2002.

[6] S. Shanmugham, "Perceptual Video Quality Measurement for Streaming Video over Mobile Networks," Master's thesis, University of Kansas, 2006.

[7] ftp://ftp.tnt.uni-hannover.de/pub/svc/testsequences

[8] JSVM 9.13.1, CVS Server, garcon.ient.rwth-aachen.de/cvs/jv [9] International Telecommunication Union (ITU) Radio Communication Sector: 'Methodology for the Subjective Assessment of the Quality of Television Pictures', ITU-R BT.500-11, 2002 [10] http://www.xrite.com, Gretag Macbeth Eye-One Display 2

[11] Z. Wang, A. Bovik, H. Sheikh, and E. Simoncelli, "Image Quality Assessment: from Error Visibility to Structural Similarity," IEEE Trans. Image Proc., 2004, pp. 600-612.

[12] D.J. Fleet, and Y. Wiess, "Optical Flow Estimation in Paragios," Handbook of Math. Models in Comp. Vis., Springer, pp. 239, 2006.

[13]J.F. Canny, "A Computational Approach to Edge Detection," IEEE Trans. Pattern Analysis and Machine Intelligence, vol. 8, pp. 679-698, 1986.

[14] C. Grigorescu, N. Petkov, M. A. Westenberg, "Contour and Boundary Detection Improved by Surround Suppression of Texture Edges," Image and Vision Computing, vol. 22, pp. 609-622, 2004.

[15] G. Papari, P. Campisi, N. Petkov, and A. Neri, "A Multiscale Approach to Contour Detection by Texture Suppression," SPIE Im. Proc.: Alg. and Syst., vol. 6064A, San Jose, CA, 2006.

[16] J. Malik, S. Belongie, T. Leung, and J. Shi, "Contour and Texture Analysis for Image Segmentation," Int. Journ. Comput. Vision, vol. 1, pp. 7-27, 2001.

[17]http://www.mathworks.com/access/helpdesk/help/toolbox/curvef it/ 